\title{
Reviewer acknowledgement Mammalia volume 83 (2019)
}

https://doi.org/10.1515/mammalia-2019-0118

The editors and the publisher wish to thank the following colleagues for their kind assistance in acting as referees for the journal this year:*

\author{
Abidu-Figueiredo, Marcelo \\ Abramov, Alexei \\ Abramson, Natalia \\ Abreu Júnior, Edson \\ Alpizar, Priscilla \\ Anile, Stefano \\ Aryal, Achyut \\ Aulia Rahman, Dede \\ Ayala-Berdon, Jorge \\ Barros, Camila \\ Bastl, Katharina \\ Benda, Petr \\ Bezerra, Alexandra M.R. \\ Bobrowiec, Paulo Estefano \\ Bogdziewicz, Michal \\ Bonvicino, Cibele \\ Boric-Bargetto, Dusan \\ Botton-Divet, Léo \\ Breviglieri, Crasso Paulo \\ Brito, Jorge \\ Brown, Peter \\ Budinski, Ivana \\ Burton, Lim K. \\ Butet, Alain \\ Cavalcanti, Gitana \\ Ceia, Ricardo \\ Chaiyarat, Rattanawat \\ Chang, Gang \\ Chetri, Madhu \\ Chevret, Pascale \\ Chiarello, Adriano \\ Cordero-Schmidt, Eugenia \\ Coyner, Brandi \\ Cristescu, Bogdan \\ Cronemberger, Cecilia \\ Crowther, Mathew \\ Cruz, Paula \\ Cruz-Salazar, Barbara \\ Cuzin, Fabrice \\ de Oliveira, João \\ De Smet, Koen \\ Deere, Nicolas \\ Delciellos, Ana C. \\ D’Elía, Guillermo \\ Deshpande, Kadambari
}

\author{
Di Bitetti, Mario \\ Di Blanco, Yamil \\ Díaz, María \\ Diaz-Varela, Emilio Rafael \\ Dinets, Vladimir \\ Dissanayake, Rajith \\ Dittmer, Drew \\ Dool, Serena E. \\ Dowler, Robert C. \\ Downs, Colleen \\ Duda, Rafaela \\ Durán, Adrián Alonso \\ Estavillo, Candelaria \\ Feijó, Anderson \\ Feng, Jiang \\ Fernández, Jesús \\ Ferreira, Guillherme \\ Figel, Joe \\ Fonturbel, Francisco \\ Freitas, Thales \\ Gable, Thomas \\ Ganem, Guila \\ Ganey, Joseph \\ Gardner, Kally \\ Gaston, Aitor \\ Geise, Lena \\ Gerard, Jean-François \\ Gerlee, Alina \\ Giannatos, Giorgos \\ Gippoliti, Spartaco \\ Gomes Rodrigues, Helder \\ Gonzalez, Susana \\ Goyal, S. P. \\ Graipel, Mauricio \\ Haigh, Amy \\ Han, Sang Hyun \\ Hannibal, Wellington \\ Harihar, Abishek \\ Hase, Kazuma \\ Hermance, John F. \\ Hernandez-Mijangos, Arturo \\ Heroldová, Marta \\ Herrera, Heitor \\ Hugot, Jean Pierre
}

\author{
Hurtado, Natali \\ Hutterer, Rainer \\ Ikeda, Takashi \\ Ivajnsic, Danijel \\ Jarnemo, Anders \\ Jenkins, Paulina \\ Jennings, Andrew \\ Jung, Thomas S. \\ Juškaitis, Rimvydas
}

Kalle, Riddhika

Kelt, Douglas

Kirby, Rebecca

Kishimoto, Ryosuke

Koprowski, John

Kotsakis, Tassos

Kowalewski, Martin

Krishna, Amitabh

Kruskop, Sergei

Lalis, Aude

Lanszki, Jozsef

Lebedev, Vladimir

Li, Baoguo

Luna, Claudia A.

Lutz, María

Machangu, Robert

Mahlaba, Themba

Mahmood, Tariq

Mahmoudi, Ahmad

Mallon, David

Martinkova, Natalia

Massara, Rodrigo

Masseti, Marco

Matos, Milene

Maul, Lutz

McLean, Bryan

Melo Azeredo, Luane M.

Mikich, Sandra

Miquelle, Dale

Mohd-Azlan, Jayasilan

Monadjem, Ara

Monterrubio-Rico, Tiberio

Montoya-Bustamante, Sebastián

Montuire, Sophie

* The time span for the list covers October 10, 2018 to October 4, 2019. 
Moreira, Danielle

Mori, Emiliano

Munroe, Karen

Nadeem, Muhammad

Newsome, Thomas

Nicolas, Violaine

Nishino, Atsuko

Nkrumah, Evans E.

Noss, Andrew

Novaes, Roberto Leonan

Ogole, Shasank

Owen, Robert

Pagh, Sussie

Panzeri, Mattia

Pardiñas, Ulyses

Patterson, Bruce

Paudel, Prakash Kumar

Pavan, Silvia

Peterkova, Renata

Pietersen, Darren

Porfirio, Grasiela

Portillo Reyes, Héctor Orlando

Prado, Joyce

Pudyatmoko, Satyawan

Puechmaille, Sébastien

Ramírez-Silva, Juan Pablo

Reljic, Slaven
Robin, V. V.

Rödel, Heiko

Rodríguez, Bernal

Rogers, Duke

Rose, Andreas

Ross, Corinna

Rostant, Luke

Rychlik, Leszek

Salgado, Ivan

Sami, Zuhair

Schiaffini, Mauro

Secco, Helio

Segall, Marion

Selonen, Vesa

Shapiro, Julie

Sharma, Lalit Kumar

Silva, Luis

Silvestre, Saulo M.

Singh, Randeep

Singleton, Grant

Soares, Fabio

Soibelzon, Esteban

Sonnweber, Ruth S.

Souza Junior, Paulo

Stukenholtz, Erin E.

Sumbera, Radim

Swift, Kaeli

Tanis, Brian

Teta, Pablo
Thibault, Martin

Timm, Robert

Tirado, Carlos

Tobler, Mathias

$\mathrm{Tu}$, Vuong Tan

Uhrin, Marcel

Vallo, Peter

Varas, Cora

Vekhnik, Viktoria

Velazco, Paúl

Velázquez, Alejandro

Vernes, Karl

Vieira, Emerson

Villalobos Chaves, David

Vogt, Kristina

Vohralik, Vladimir

Wang, Tianming

Warburton, Elizabeth

Wechuli, David B.

Weldone, Andy

Wong, Seth

Wordley, Claire

Wronski, Torsten

Yahnke, Christopher J.

Zukal, Jan 